\title{
Feocromocitoma: uma causa rara de hipertensão arterial na infância
}

\author{
Pheochromocytoma: a rare cause of hypertension in childhood
}

Flávia Carolina D. Georgetti ${ }^{1}$, Gisela de Rezende Eugênio ${ }^{1}$

\section{RESUMO}

Objetivo: Relatar o caso de um adolescente com feocromocitoma, uma causa rara de hipertensão arterial na infância.

Descrição: Adolescente internado em unidade de terapia intensiva infantil em decorrência de emergência hipertensiva, conseqüente à presença de feocromocitoma em adrenal esquerda, diagnosticado por meio de tomografia computadorizada do abdome e pela dosagem de adrenalina e noradrenalina urinárias. O paciente foi submetido à adrenalectomia esquerda, após o uso de $\alpha$-bloqueador para controle do quadro hipertensivo. $\mathrm{O}$ anatomopatológico confirmou o diagnóstico do tumor. No pós-operatório, o paciente permaneceu estável, possibilitando a suspensão dos anti-hipertensivos.

Comentários: Os feocromocitomas são tumores capazes de produzir catecolaminas, especialmente adrenalina e/ou noradrenalina. Cerca de 85 a 95\% dos tumores são únicos, benignos e encontrados na medula adrenal. O feocromocitoma é um tumor de incidência rara e apenas 10 a 20\% ocorrem na infância, representando uma causa rara de hipertensão arterial. Esta última é um sinal freqüente na maioria das crianças (80\%), podendo ser acompanhada por cefaléia e sudorese. A encefalopatia hipertensiva consiste em uma forma de apresentação excepcional da doença. O diagnóstico pode ser realizado, na maioria dos casos, pela tomografia de abdome e pela dosagem das catecolaminas e seus metabólitos produzidos pelo tumor. O tratamento de escolha consiste na ressecção completa do tumor após o preparo farmacológico do paciente com o uso de $\alpha$-bloqueador. No pós-operatório, a maioria dos pacientes evolui com controle do quadro de hipertensão arterial.

Palavras-chave: feocromocitoma; hipertensão; medula supra-renal; criança.

\section{ABSTRACT}

Objective: Report an adolescent with pheochromocytoma, a rare cause of hypertension in childhood.

Case description: Adolescent admitted to the pediatric intensive care unit due to hypertension, secondary to the presence of pheochromocytoma on the left adrenal. Diagnosis of the pheochromocytoma was made by abdominal computed tomography scan and by measuring urinary epinephrine and norepinephrine. The patient underwent excision of the left adrenal, after controlling blood pressure levels with an $\alpha$-blocker drug. The histological analysis confirmed the diagnosis. After the surgery, the patient remained under control and the use of anti-hypertensive medication was stopped.

Comments: Pheochromocytomas are catecholaminesecreting tumors, specially epinephrine and norepinephrine. Almost 85 to $95 \%$ of the tumors are single, benign and found on adrenal medulla. Pheochromocytoma is a rare tumor - only 10 to $20 \%$ occur in childhood - and can cause hypertension. Hypertension is found in the majority of pediatric patients (80\%), and may occur associated to headache and sweating. Hypertensive encephalopathy is very rare. The diagnosis can be done, in most cases, by computed tomography scans of the abdomen and by measuring levels of catecholamines and their metabolites produced by the tumor. The treatment of choice is the complete resection of the tumor after the use of an $\alpha$-blocker medication. After the surgery, the majority of patients recover from hypertension.

Key-words: pheochromocytoma; hypertension; adrenal medulla; child.
${ }^{1}$ Especialista em Terapia Intensiva Pediátrica e professora colaboradora do Departamento de Pediatria da Faculdade de Medicina de Jundiaí (FMJ)

Endereço para correspondência:

Flávia Carolina D. Georgetti
Avenida Francisco Pereira de Castro, 761, apto. 101 - Anhangabaú

CEP 13208-110 - Jundiaí/SP

E-mail: fgeorgetti@uol.com.br

Recebido em: 22/2/2007

Aprovado em: 18/6/2007 


\section{Introdução}

Os feocromocitomas são tumores originados nas células cromafins do eixo simpático adrenomedular, produtores autônomos de catecolaminas, especialmente adrenalina e/ou noradrenalina ${ }^{(1)}$. Logo após o nascimento, a maioria das células cromafins, com exceção das células da medula adrenal, se degenera, de modo que 85 a $95 \%$ dos feocromocitomas são encontrados na medula adrenal. Os tumores também podem ter origem fora da medula adrenal, sendo denominados paragangliomas funcionais. Ocorrem mais freqüentemente no órgão de Zuckerkandl (75\%), mas também podem ser encontrados no tórax, abdome, pelve, mediastino e pescoço ${ }^{(2,3)}$.

Originalmente descrito por Frankel em 1886, o feocromocitoma é um tumor de incidência rara (dois a oito casos para cada 1.000.000 de habitantes/ano), com pico de incidência entre a terceira e a quinta décadas de $v_{i d a}{ }^{(3,4)}$. Apenas 10 a $20 \%$ dos casos ocorrem na infância ${ }^{(5-8)}$.

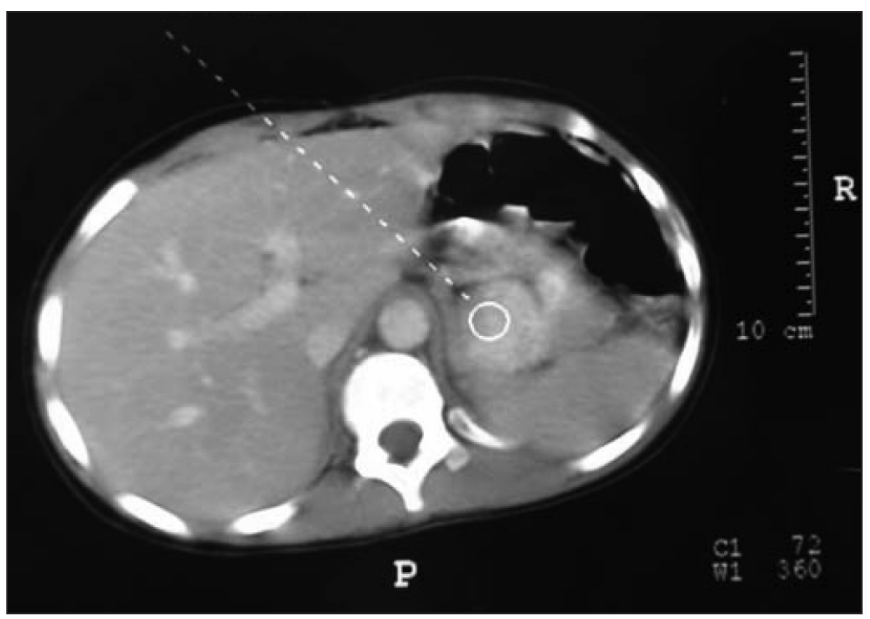

Figura 1-Tomografia computadorizada de abdome demonstrando a presença de uma massa em adrenal esquerda

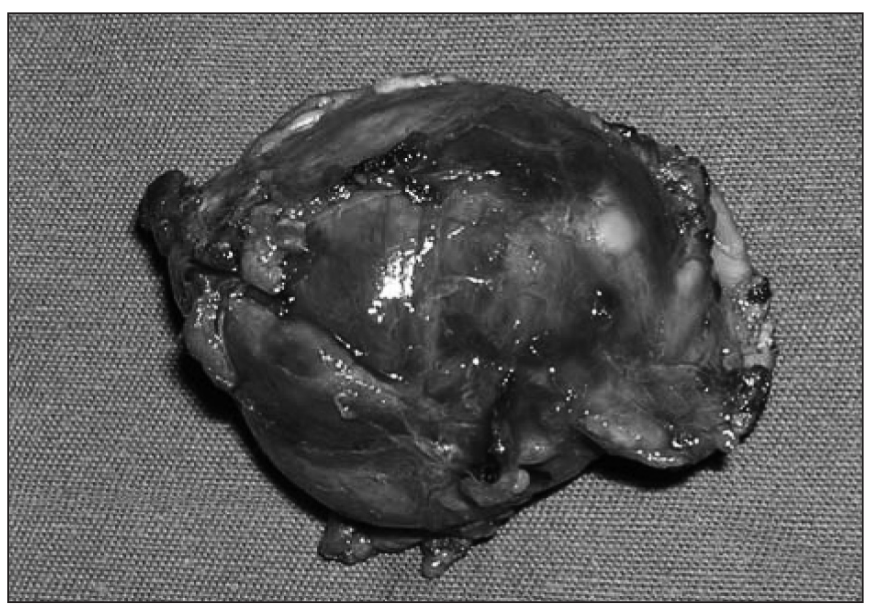

Figura 2 - Peça cirúrgica que confirmou o diagnóstico de feocromocitoma em adrenal esquerda
Cerca de $90 \%$ dos tumores são únicos e benignos. Tumores múltiplos são encontrados com maior freqüência em casos de feocromocitoma de origem familiar. Em 10 a 15\% dos casos, os feocromocitomas são herdados como traço autossômico dominante e podem ser o primeiro sinal da presença de neoplasia endócrina múltipla tipo 2 ou de síndromes neuroectodérmicas (neurofibromatose tipo I, doença de von Hippel-Lindau). A neoplasia endócrina múltipla tipo $2 \mathrm{~A}$ é caracterizada pela presença de feocromocitoma, carcinoma medular de tireóide e hiperparatireoidismo. Embora o diagnóstico do feocromocitoma e do carcinoma medular de tireóide possa ser realizado em diferentes épocas, os dois tumores são diagnosticados simultaneamente em cerca de 70\% dos casos. A neoplasia endócrina múltipla tipo $2 \mathrm{~B}$, por sua vez, consiste em feocromocitoma, carcinoma medular de tireóide, neuromas de mucosas, ganglioneuromatose e aspecto marfanóide ${ }^{(3,9)}$.

O feocromocitoma representa causa potencialmente letal de hipertensão arterial, respondendo por cerca de $1 \%$ dos pacientes que desenvolvem hipertensão arterial na infância ou na adolescência ${ }^{(10)}$. Os autores descrevem o caso de um feocromocitoma diagnosticado em um menino de 12 anos, que desenvolveu um quadro de emergência hipertensiva.

\section{Relato do caso}

Criança do gênero masculino, 12 anos, internado na Unidade de Terapia Intensiva Infantil do Hospital Universitário da Faculdade de Medicina de Jundiaí em decorrência de crise convulsiva tônico-clônica generalizada, com pressão arterial de 200x120mmHg. Havia antecedente de cefaléia há dois anos, parestesia em face e em membros superior e inferior direito. $\mathrm{O}$ paciente estava comatoso à admissão e foi submetido à intubação orotraqueal e assistência ventilatória. Iniciado o tratamento para emergência hipertensiva com nitroprussiato de sódio. $\mathrm{O}$ raio- $\mathrm{X}$ de tórax não revelou aumento da área cardíaca e os valores de pressão arterial foram semelhantes, quando aferidos nos quatro membros. O paciente não apresentava anormalidades na função renal e o exame de urina não revelou a presença de proteínas.

Realizada tomografia computadorizada (TC) de crânio que demonstrou edema cerebral difuso. Nos primeiros três dias, o paciente evoluiu com controle dos níveis pressóricos em uso de nitroprussiato de sódio e recuperação do nível de consciência, sendo possível a extubação. No quarto dia de internação, a ultra-sonografia de abdome revelou a presença de uma massa hipoecogênica em topografia de adrenal esquerda. Iniciado o tratamento para hipertensão arterial sistêmica por meio do uso de prazosina, um bloqueador $\alpha-1$ seletivo pelo provável diagnóstico de feocromocitoma. A TC de abdome confirmou a 
presença de uma massa em adrenal esquerda, com $4,0 \times 3,8 \mathrm{~cm}$ (Figura 1). A dosagem de catecolaminas urinárias resultou em valores elevados para adrenalina e noradrenalina.

Houve controle do quadro de hipertensão arterial com o uso do $\alpha$-bloqueador, sendo suspenso o nitroprussiato de sódio após cinco dias. No quinto dia em uso de prazosina, foi introduzido o propranolol. No $12^{\circ}$ dia de internação, estável em uso de prazosina há uma semana, o paciente foi submetido à adrenalectomia esquerda.

A análise da peça cirúrgica confirmou o diagnóstico de feocromocitoma. O tumor de medidas $3,5 \times 3,0 \times 3,0 \mathrm{~cm}$, encontrado na adrenal esquerda, estava encapsulado e apresentava áreas focais de hemorragia e necrose. Não havia invasão capsular ou vascular e as margens cirúrgicas estavam livres. Não foram identificados sinais histológicos de malignidade (Figura 2). No pós-operatório, o paciente evoluiu com normalização dos níveis pressóricos, permitindo a suspensão da medicação anti-hipertensiva. O paciente permaneceu estável e se encontra sob acompanhamento ambulatorial.

\section{Discussão}

Os principais sinais e sintomas apresentados pelos portadores de feocromocitomas são decorrentes da liberação excessiva de catecolaminas. Cerca de $90 \%$ dos pacientes apresentam hipertensão arterial, freqüentemente resistente ao tratamento convencional, mas podendo responder ao uso de $\alpha$-adrenérgicos, bloqueadores dos canais de cálcio e nitroprussiato de sódio. Em 25 a 40\% dos casos, os feocromocitomas manifestam-se na forma de paroxismos hipertensivos. Especialmente nas crianças, a hipertensão arterial é um sinal constante, sendo encontrada em até $80 \%$ dos casos. Proteinúria, retinopatia e encefalopatia hipertensiva, a exemplo do ocorrido no caso relatado, são ocasionalmente observados como complicações decorrentes da hipertensão arterial ${ }^{(3,4,11)}$.

Cefaléia (72\%), sudorese (69\%) e palpitações (51\%) constituem os principais sintomas associados à hipertensão arterial em pacientes com feocromocitoma. O encontro desta tríade de sintomas em um paciente hipertenso está relacionado a índices de sensibilidade e especificidade de 90,9 e 93,8\%, respectivamente, no diagnóstico de feocromocitoma ${ }^{(12)}$. Sintomas como ansiedade, rubor facial, palidez cutânea, náuseas e vômitos também podem ser observados em portadores deste tumor.

Os pacientes podem apresentar angina e infarto agudo do miocárdio, decorrentes do espasmo coronariano, pelo aumento das catecolaminas e do neuropeptídeo Y, aumento da agregação plaquetária e do consumo de oxigênio ${ }^{(3,4)}$. O feocromocitoma pode se manifestar como edema agudo de pulmão, decorrente de insuficiência cardíaca esquerda ou de alterações na permeabilidade capilar mediada por catecolaminas e outros peptídeos ${ }^{(13)}$.
Cerca de $40 \%$ dos portadores de feocromocitomas desenvolvem hipotensão ortostática e/ou choque, decorrente da redução do volume plasmático e da exacerbação dos reflexos posturais pela ação das catecolaminas em receptores présinápticos $\alpha$-2-adrenérgicos, resultando em prejuízo das respostas dependentes da noradrenalina e/ou redução da sensibilidade dos receptores em nível vascular ${ }^{(4,11,13)}$. Arritmias cardíacas, como fibrilação atrial e ventricular, consistem em uma causa freqüente de morte súbita em portadores de feocromocitoma, especialmente durante o período de anestesia e/ou de ressecção tumoral. Acidentes vasculares cerebrais isquêmicos ou hemorrágicos também podem ocorrer ${ }^{(11,13)}$.

Os pacientes podem apresentar sudorese intensa, intolerância ao calor, aumento da temperatura corporal e perda ponderal. Intolerância à glicose ou mesmo diabetes melito podem decorrer do efeito inibitório das catecolaminas sobre a liberação de insulina. Mais raramente, observa-se hipercalcemia resultante da ação das catecolaminas nas paratireóides ou diretamente sobre os ossos ${ }^{(4,11,13,14)}$. Portadores de feocromocitoma podem mostrar redução da motilidade do trato gastrintestinal, resultando em obstipação intestinal. Eventualmente, dor abdominal, decorrente de isquemia em fígado ou rins, pode ser o principal sintoma da doença ${ }^{(15)}$. A dor abdominal também pode decorrer de necrose do próprio tumor ${ }^{(12)}$. No entanto, alguns pacientes podem apresentar sintomas tênues ou permanecer assintomáticos durante muito tempo, sobretudo nos casos de síndromes familiares ou naqueles em que o diagnóstico é realizado de maneira acidental, por meio de exames de imagem ${ }^{(6,16)}$.

O diagnóstico laboratorial baseia-se na demonstração da produção das catecolaminas ou de seus metabólitos pelo tumor. Em nosso meio, as dosagens de ácido vanilmandélico (VMA), adrenalina e noradrenalina (no plasma e na urina), bem como de metanefrinas e normetanefrinas (na urina) são os testes disponíveis para o diagnóstico de feocromocitoma.

Uma vez que a secreção de catecolaminas pelo tumor pode ser intermitente, dosagens isoladas de adrenalina e noradrenalina no plasma, bem como de VMA (valor preditivo positivo de $72 \%$ ), não estão indicadas como instrumento único para o diagnóstico de feocromocitoma. Apenas valores plasmáticos muito elevados (acima de 2000pg/mL) de adrenalina e noradrenalina podem ser úteis no diagnóstico deste tipo de tumor. Em estudo realizado por $\mathrm{Bravo}^{(13)}$, cerca de $30 \%$ dos portadores de feocromocitoma apresentaram níveis plasmáticos de catecolaminas semelhantes aos de hipertensos essenciais. Em relação ao VMA, a dosagem simultânea de metanefrina aumenta a sensibilidade do teste, permitindo que até $98 \%$ dos tumores sejam diagnosticados ${ }^{(17-22)}$.

Quando dosadas na urina, no entanto, a adrenalina e a noradrenalina consistem em instrumentos sensíveis e específicos 
para o diagnóstico de feocromocitoma. Graham ${ }^{(18)}$ encontrou sensibilidade de $100 \%$ e especificidade de $97 \%$ na dosagem simultânea das duas catecolaminas. No caso descrito, a análise de uma amostra de urina de 24 horas resultou em valores elevados de ambas as catecolaminas.

Embora a produção de catecolaminas pelo tumor possa ser intermitente, sua metabolização ocorre continuamente; desta forma a dosagem de metanefrinas corresponde ao método de melhor valor preditivo positivo para o diagnóstico de feocromocitomas ${ }^{(23-25)}$. Metanefrina e normetanefrina, quando dosadas simultaneamente na urina, consistem em um instrumento diagnóstico com sensibilidade de $100 \%$ e especificidade de $98 \%$. Quando dosadas separadamente, a metanefrina tem sensibilidade de $90 \%$ e a normetanefrina, de $70 \%{ }^{(18)}$.

Uma vez detectada a presença de um feocromocitoma por meio dos testes bioquímicos, a determinação da localização e da extensão do tumor são imprescindíveis. Embora a ultra-sonografia possa detectar grande parte dos feocromocitomas, a tomografia computadorizada consiste em um dos principais métodos de localização deste tipo de tumor, com sensibilidade de aproximadamente 93 a $100 \%$ no diagnóstico de massas adrenais com mais de $1 \mathrm{~cm}$ e de cerca de $90 \%$ para os tumores extra-adrenais ${ }^{(3,13,26,27)}$. Os índices de sensibilidade da ressonância magnética em relação ao diagnóstico de feocromocitomas são bastante semelhantes aos apresentados pela tomografia ${ }^{(27)}$. Infelizmente, no entanto, a especificidade destes exames é baixa, podendo ser de apenas $50 \%$ (28).

$\mathrm{O}$ mapeamento com ${ }^{131} \mathrm{I}$ metaiodobenzilguanidina $\left({ }^{131} \mathrm{I}\right.$ MIBG) consiste, por sua vez, em exame de alta especificidade (95 a 100\%) para o diagnóstico de feocromocitomas, estando indicado sobretudo nos casos de recidiva tumoral e para localizar feocromocitomas extra-adrenais e metástases ${ }^{(20,28)}$.

O tratamento de escolha para os feocromocitomas é a remoção cirúrgica do tumor, sendo necessário um adequado bloqueio hormonal no pré-operatório. Bloqueadores $\alpha$-adrenérgicos para controle dos paroxismos devem ser iniciados sete a dez dias antes do ato cirúrgico ${ }^{(4,29,30)}$.

Embora a fenoxibenzamina seja o $\alpha$-bloqueador classicamente utilizado no tratamento do feocromocitoma, essa medicação não é comercializada em nosso país. A prazosina, um bloqueador $\alpha-1$ seletivo, constitui uma alternativa para o bloqueio $\alpha$-adrenérgico destes pacientes. Em crianças, a dose recomendada é de 25 a $150 \mathrm{mcg} / \mathrm{kg} / \mathrm{dia}$, com aumento progressivo a partir de uma dose teste de $5 \mathrm{mcg} / \mathrm{kg}$ até o máximo de $12 \mathrm{mg} / \mathrm{dia}$. O principal efeito colateral é a hipotensão postural, dose dependente e mais freqüente na fase inicial de administração da medicação ${ }^{(30-32)}$. No caso relatado, observou-se rápida resposta ao uso da prazosina, possibilitando a suspensão do uso do nitroprussiato de sódio, potencialmente tóxico pelo possível acúmulo de cianeto.
A fentolamina, outro bloqueador $\alpha$-adrenérgico, também é usualmente utilizada em pacientes com feocromocitoma. $\mathrm{O}$ efeito da medicação resulta do bloqueio da ação das catecolaminas circulantes sobre os receptores alfa- 1 e alfa- 2 adrenérgicos. A fentolamina, freqüentemente administrada aos pacientes no préoperatório imediato ou durante a ressecção do tumor, é capaz de prevenir ou controlar os paroxismos de hipertensão decorrentes do ato anestésico ou da manipulação do tumor. Em crianças, a dose de $1 \mathrm{mg} / \mathrm{kg}$ deve ser administrada uma a duas horas antes da cirurgia, podendo ser repetida durante a mesma, se necessário ${ }^{(33)}$.

O controle da taquicardia, decorrente dos elevados níveis de adrenalina e noradrenalina circulantes e do bloqueio dos receptores $\alpha$-adrenérgicos, pode requerer o uso de $\beta$-bloqueadores, como o propranolol ou metoprolol, no pré-operatório. Uma vez que o uso isolado de $\beta$-bloqueadores em pacientes com feocromocitoma tem sido relacionado a episódios de aumento paradoxal da pressão arterial, sua administração deve estar condicionada ao uso prévio de um $\alpha$-bloqueador ${ }^{(32,33)}$.

Todo feocromocitoma deve ser abordado como potencialmente maligno, sendo preconizada a ressecção do tumor com a cápsula intacta. Nos últimos anos, a adrenalectomia laparoscópica é recomendada como a modalidade de escolha para o tratamento do feocromocitoma, principalmente em caso de lesões menores do que $8 \mathrm{~cm}^{(16,34,35)}$. A cirurgia aberta ainda é recomendada diante de lesões grandes ou malignas, com suspeita de invasão local ou doença metástatica ${ }^{(16,36)}$.

Alguns autores acreditam que pacientes com feocromocitomas decorrentes de síndromes familiares, como a doença de von Hippel-Lindau ou a neoplasia endócrina múltipla, devem ser submetidos à adrenalectomia bilateral, uma vez que $90 \%$ apresentam tumores bilaterais ou hiperplasia da medula adrenal, um precursor do tumor. No entanto, nos pacientes com feocromocitomas não familiares, nos quais a incidência de bilateralidade, hiperplasia adrenal e recorrência é bem menor, a adrenalectomia total unilateral é a melhor abordagem cirúrgica ${ }^{(37)}$.

No pós-operatório, a redução do estímulo dos receptores adrenérgicos pelas catecolaminas, a depleção volumétrica e o efeito residual dos $\alpha$-bloqueadores podem provocar hipotensão. Por outro lado, o excesso de reservas de catecolaminas e a instabilidade autonômica podem determinar episódios de hipertensão no pós-operatório imediato. A persistência da hipertensão arterial ou sua recorrência podem decorrer de um tumor residual ou da presença de metástases ${ }^{(13)}$. A glicemia deve ser monitorizada no pós-operatório, devido ao risco de hipoglicemia por hiperinsulinemia causada pela suspensão do efeito supressor das catecolaminas sobre a liberação insulínica ${ }^{(32)}$.

Diante de um preparo pré-operatório adequado e da abordagem do paciente por cirurgiões e anestesistas experientes, bem 
como de um manejo adequado no pós-operatório, o índice de sucesso no tratamento do feocromocitoma é alto (98\%). A ressecção do tumor resulta em controle da hipertensão arterial na maioria dos casos, como observado no paciente aqui descrito.

Em uma série de crianças acompanhadas por Ciftci et al ${ }^{(37)}$, a incidência de recorrência foi de aproximadamente $22 \%$ em tumores de aspecto benigno e de cerca de $50 \%$ naqueles com características malignas. A taxa de sobrevida em cinco anos para pacientes portadores de feocromocitomas benignos submetidos ao tratamento cirúrgico pode ser superior a $95 \%$. Para os portadores de tumores malignos, esse índice pode ser inferior a $50 \%$. Felizmente, no entanto, a taxa de malignidade dos

\section{Referências bibliográficas}

1. Gerlo EAM, Sevens C. Urinary and plasma catecholamines and urinary catecholamine metabolites in pheochromocytoma: diagnostic value in 19 cases. Clin Chem 1994:40:250-6.

2. Whalen RK, Althausen AF, Daniels GH. Extra-adrenal pheochromocytoma. J Urol 1992;147: 1-10.

3. Hall AS, Ball SG. Phaeochromocytoma. Neth J Med 1993;43:S29-38.

4. Lansberg L, Young JB. Catecholamines and the adrenal medulla. In: Wilson JD, Foster DW, editors. Textbook of endocrinology. $8^{\text {th }}$ ed. Philadelphia: Saunders; 1993. p. 621-705.

5. Townsend CB, Evers M, Beauchamp RD. The pituitary and adrenal glands. In: Townsend CM, Beauchamp RD, Evers BM, Mattox KL, editors. Sabiston textbook of surgery. $17^{\text {th }}$ ed. Philadelphia: Elsevier 2004. p. 1052-89.

6. Yeo H, Roman S. Pheochromocytoma and functional paraganglioma. Curr Opin Oncol 2005;17:13-8.

7. Ein SH, Shandling B, Wesson D, Filler R. Recurrent pheochromocytomas in children. J Pediatr Surg 1990;25:1063-5.

8. Fonkalsrud EW. Pheochromocytoma in childhood. Prog Pediatr Surg 1991;26:103-11.

9. Casanova S, Rosenberg-Bourgin M, Farkas D, Calmettes C, Feingold N, Heshmati HM et al. Phaeochromocytoma in multiple endocrine neoplasia type 2A: survey of 100 cases. Clin Endocrinol 1993;38:531-7.

10. Pickard JL, Ross G, Silver D. Coexisting extraadrenal pheochromocytoma and renal artery stenosis: a case report and review of the pathophysiology. J Pediatr Surg 1995;30:1613-5.

11. Benovwitz NL. Pheochromocytoma. Adv Intern Med 1990;35:195-220.

12. Bravo EL, Tagle R. Pheochromocytoma: state-of-the-art and future prospects. Endocr Rev 2003;24:539-53.

13. Bravo EL. Pheochromocytoma: new concepts and future trends. Kidney Int 1991;40:544-56.

14. Deal JE, Sever PS, Barratt TM, Dillon MJ. Phaeochromocytoma - investigation and management of 10 cases. Arch Dis Child 1990;65:269-74.

15. Levine C, Levine E. Renal infarction associated with an extraadrenal pheochromocytoma. J Pediatr Surg 1992;27:657-9.

16. Roman S. Pheochromocytoma and functional paraganglioma. Curr Opin Oncol 2004;16: 8-12.

17. Peaston RT, Lai LC. Biochemical detection of phaeochromocytoma: should we still be measuring urinary HMMA? J Clin Pathol 1993;46:734-7.

18. Graham PE, Smythe GA, Edwards GA, Lazarus L. Laboratory diagnosis of phaeochromocytoma: which analytes should we measure? Ann Clin Biochem 1993;30:129-34.

19. Sawka AM, Jaeschke R, Singh RJ, Young WF Jr. A comparison of biochemical tests for pheochromocytoma: measurement of fractionated plasma metanephrines compared with the combination of 24-hour urinary metanephrines and catecholamines. J Clin Endocrinol Metab 2003;88:553-8.

20. Bravo EL. Evolving concepts in the pathophysiology, diagnosis and treatment of pheochromocytoma. Endocr Rev 1994;15:356-68.

21. Stringel G, Ein SH, Creighton R, Daneman D, Howard N, Filler RM. Pheochromocytoma in children--an update. J Pediatr Surg 1980;15:496-500. feocromocitomas adrenais em adultos varia entre 8,3 e 13,1\% e, em crianças, este taxa parece ser ainda menor ${ }^{(20,22,38)}$.

Embora bastante raros, os feocromocitomas consistem em uma causa importante de hipertensão arterial na faixa etária pediátrica, devendo ser investigados e prontamente tratados em crianças e adolescentes hipertensos. Diante do padrão heterogêneo de secreção de catecolaminas pelo tumor, nenhum ensaio bioquímico deve ser usado isoladamente como instrumento diagnóstico. A localização do tumor e a determinação do seu potencial de malignidade contribuem para a escolha da via cirúrgica. O diagnóstico precoce e a ressecção completa do tumor são os principais fatores determinantes de um melhor prognóstico.

22. Sawin RS. Functioning adrenal neoplasms. Semin Pediatr Surg 1997; 6:156-63.

23. Lenders JW, Pacak K, Walther MM, Linehan WM, Mannelli M, Friberg $P$ et al. Biochemical diagnosis of pheochromocytoma: which test is best? JAMA 2002;287:1427-34.

24. Eisenhofer G, Goldstein DS, Walther MM, Friberg P, Lenders JW, Keiser HR et al. Biochemical diagnosis of pheochromocytoma: how to distinguish truefrom false-positive test results. J Clin Endocrinol Metab 2003;88:2656-66.

25. Lenders JW, Pacak K, Eisenhofer G. New advances in the biochemical diagnosis of pheochromocytoma: moving beyond catecholamines. Ann N Y Acad Sci 2002;970:29-40.

26. Plouin PF, Chatellier G, Rougeot MA, Duclos JM, Pagny JY, Corvol P et al. Recent developments in pheochromocytoma diagnosis and imaging. Adv Nephrol Necker Hosp 1988;17:275-86.

27. Ilias I, Pacak K. Current approaches and recommended algorithm for the diagnosis Iocalization of pheochromocytoma. J Clin Endocrinol Metab 2004;89:479-91.

28. Maurea S, Cuocolo A, Reynolds JC, Tumeh SS, Begley MG, Linehan WM et al. lodine 131 metaiodobenzylguanidine scintigraphy in preoperative and postoperative evaluation of paragangliomas: comparison with CT and MRI. J Nucl Med 1993;34:173-9.

29. Kaplan NM. Endocrine hypertension. In: Wilson JD, Foster DW, editors. Textbook of endocrinology. $8^{\text {th }}$ ed. Philadelphia: Saunders;1993. p. 7-31.

30. Colucci WS. Alpha-adrenergic receptor blockade with prazosin. Consideration of hypertension, heart failure, and potential new applications. Ann Intern Med 1982;97:67-77.

31. Cubeddu LX, Zarate NA, Rosales CB, Zschaeck DW. Prazosin and propranolol in preoperative management of pheochromocytoma. Clin Pharmacol Ther 1982;32:156-60

32. Faiçal S, Shiota D. Feocromocitoma: atualização diagnóstica e terapêutica. Rev Assoc Med Bras 1997;43:237-44.

33. Drugs.com - Drug information online [homepage na Internet]. Micromedex $^{\mathrm{TM}}$, Facts \& Comparisons ${ }^{\mathrm{TM}}$ and Multum ${ }^{\mathrm{TM}}$; c2000-2007 [atualizado em 7 de junho de 2007; citado em 18 de junho de 2007]. Drugs.com; [phentolamine; cerca de 1 tela]. Disponível em: http://www.drugs.com/ppa/phentolamine.html

34. Matsuda T, Murota T, Oguchi N, Kawa G, Muguruma K. Laparoscopic adrenalectomy for pheochromocytoma: a literature review. Biomed Pharmacother 2002;56:S132-8.

35. Fletcher DR, Beiles CB, Hardy KJ. Laparoscopic adrenalectomy. Aust NZ J Surg 1994;64:427-30.

36. Orchard T, Grant CS, van Heerden JA, Weaver A. Pheochromocytoma--continuing evolution of surgical therapy. Surgery 1993;114:1153-9.

37. Ciftci AO, Tanyel FC, Senocak ME, Büyükpamukçu N. Pheochromocytoma in children. J Pediatr Surg 2001;36:447-52.

38. Goldstein RE, O'Neill JA, Holcomb GW, Morgan WM, Neblet WW, Oates JA et al. Clinical experience over 48 years with pheochromocytoma. Ann Surg 1999;229:755-66. 